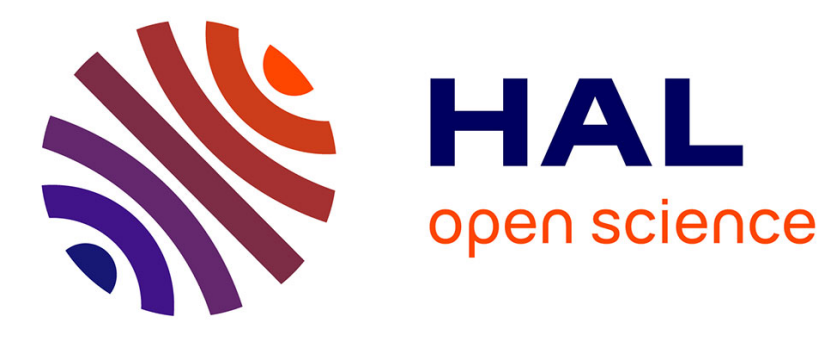

\title{
Dynamical systems that compute time-optimal switchings
}

Frédéric Grognard, Rodolphe Sepulchre, Jose de Dona

\section{To cite this version:}

Frédéric Grognard, Rodolphe Sepulchre, Jose de Dona. Dynamical systems that compute time-optimal switchings. IFAC NOLCOS 2001 Symposium, Jul 2002, Saint Petersburg, Russia. hal-01091608

\section{HAL Id: hal-01091608 \\ https://hal.inria.fr/hal-01091608}

Submitted on 5 Dec 2014

HAL is a multi-disciplinary open access archive for the deposit and dissemination of scientific research documents, whether they are published or not. The documents may come from teaching and research institutions in France or abroad, or from public or private research centers.
L'archive ouverte pluridisciplinaire HAL, est destinée au dépôt et à la diffusion de documents scientifiques de niveau recherche, publiés ou non, émanant des établissements d'enseignement et de recherche français ou étrangers, des laboratoires publics ou privés. 


\title{
Dynamical systems that compute time-optimal switchings
}

\author{
F. Grognard ${ }^{\dagger}$, R. Sepulchre ${ }^{\ddagger}, \dagger$, J. De Dona ${ }^{\ddagger}$ \\ ${ }^{\dagger}$ Center for Systems Engineering and Applied Mechanics \\ Université catholique de Louvain, \\ Av. G. Lemaitre 4, B1348 Louvain-La-Neuve, Belgium. \\ $\ddagger$ Institut Montefiore, B28 \\ Université de Liège, \\ B4000 Liège Sart-Tilman, Belgium. \\ email: \{grognard $\},\{$ sepulchre $\},\{$ dedona $\} @$ montefiore.ulg.ac.be
}

Submitted to NOLCOS 2001

\begin{abstract}
It is well known that the minimum time bounded control of linear systems is generically bang-bang and that the number of switchings does not exceed the dimension of the system if the eigenvalues of the system matrix are real. We propose a synthesis method for such problems based on dynamical systems that 'compute' the optimal sequence of switching times.
\end{abstract}

\section{Introduction}

This paper addresses the classical synthesis problem of time-optimal control laws for a single-input linear system $\dot{z}=A z+b v$ subject to the bounded input constraint $|v| \leq 1$. We consider the situation of a controllable pair $(A, b)$ and assume that all the eigenvalues of $A$ are real. In this case, it is well-known from the maximum principle that the optimal control $u(t)$ that steers an initial state $z(0)=z_{0}$ to a final state $z(T)=z_{f}$ in minimum time is bang-bang, i.e. switches between the extreme values $u=+1$ and $u=-1$, and that the number of switchings is at most $n-1$, where $n$ is the dimension of the system (Lee \& Markus 1967). In other words, the computation of the time-optimal control reduces to the computation of the optimal sequence of switchings times $t_{0}=0, t_{1}, \ldots, t_{n}=T$, or equivalently, the optimal sequence of time-intervals $\bar{x}_{1}=t_{1}-t_{0}, \bar{x}_{2}=t_{2}-t_{1}, \ldots, \bar{x}_{n}=t_{n}-t_{n-1}$. 
In this paper, we construct continuous time-systems $\dot{x}=f(x)$ which 'produce' the optimal sequence $\bar{x}=\left(\bar{x}_{1}, \ldots, \bar{x}_{n}\right)^{T}$, in the sense that they possess a unique equilibrium at $x=\bar{x}$ and that this equilibrium is asymptotically stable. The systems are positive, i.e. they are only defined in the open positive orthant $\mathcal{O}^{+}=\left\{x \mid x_{i}>0, i=1, \cdots, n\right\}$ only and the dynamics leave this open set invariant. Our main result shows that, under proper time-scale decomposition, the convergence of solutions to the desired equilibrium $x=\bar{x}$ can be enforced in a rather transparent way, through a sequence of nested invariant manifolds. This manifold structure connects the convergence properties of the algorithm to the geometry of the time-optimal control problem and supports the excellent convergent properties that are observed in simulations, even when the time scales are no longer enforced.

Discrete-time versions of the algorithms investigated in this paper include two algorithms previously proposed in (Yastreboff 1969a)-(Yastreboff 1969b) and, more recently, in (De Doná 2000). No convergence analysis is proposed in those references but simulations suggest strong (and in fact global) convergence properties. The continuous-time algorithm is more amenable to a time-scale decomposition analysis but the geometry displayed in the present paper is roughly retained in discrete-time versions as well.

It is important to notice that the algorithms discussed in the present paper are based on a particular heuristics, to be explained in Section 2. In that respect, they differ from the realm of gradient-based iterative methods for the synthesis of time-optimal controllers, that date back almost to the starting point of the time-optimal control problem itself (Bellman et al. 1956, Desoer 1959). These gradient methods typically iterate on the adjoint initial or final state together with the time of response (see for instance (Neustadt 1960, Ho 1962, Fadden \& Gilbert 1964, Gilbert 1964)). It is known that these methods are, in general, sensitive to the starting condition (initial guess) and have poor convergence properties. This is in contrast with the convergence properties displayed in the present paper.

A 'modern' motivation for studying iterative methods for the synthesis of optimal control laws is in their potential implementation in a recedinghorizon fashion, so as to make corrections to the control policy when the plant is affected by inaccuracies or by external disturbances. With the current computing power and with the maturity reached by the theory of recedinghorizon control (Mayne et al. 2000), there is a renewed interest in efficient algorithms for the synthesis of optimal control laws.

The remainder of the paper is organized as follows. In Section 2 we provide some background and the heuristics of the iterative methods discussed in this paper. In Section 3 we analyze the convergence properties of a particular continuous-time version of the algorithm based on a timescale decomposition of the $n$-dimensional system into $n$ scalar subsystems. Concluding remarks are presented in Section 4. 


\section{A heuristics for the iterative computation of op- timal switching times}

Consider the linear system

$$
\dot{z}=A z+b v
$$

where $z \in \mathbb{R}^{n}$ is the state and $v \in \mathbb{R}$ is the input which is constrained to $|v| \leq 1$. We assume that the system is normal, i.e., that the pair $(A, b)$ is controllable (see, e.g., Lee \& Markus 1967). We further assume that the eigenvalues of matrix $A$ are real. The problem is to find the control function $v^{0}(t)$ that steers the trajectory from a specified initial state $z(0)=z_{0}$ to a specified final state here chosen to be the origin $z\left(t_{f}\right)=z_{f}=0$ in minimum time $t_{f}^{0}$. We assume that $z_{0}$ is in the null-controllable set and not on a switching surface of the time-optimal controller. From the maximum principle it can be concluded (Lee \& Markus 1967) that the time-optimal control is 'bang-bang' (i.e., piecewise constant, with each component assuming only the values \pm 1 ) and it has exactly $n-1$ switchings; and, any 'bang-bang' policy with at most $n-1$ switchings is optimal in the sense that whatever state the plant reaches using such a control, it does so in optimal time. Further references for these properties can be found in (Sontag 1998).

As a consequence of these properties, we can restrict our search for the optimal control among the steering controls that are defined by a sequence of $n$ time intervals $x_{i}=t_{i}-t_{i-1}$ and the corresponding sequence of constant control values $u_{i}$. This class of piecewise constant controls (see Figure 1) is thus characterized by two vectors, namely the vector of time intervals

$$
x=\left(\begin{array}{l}
x_{1} \\
\ldots \\
x_{n}
\end{array}\right)
$$

and the vector of control values

$$
u=\left(\begin{array}{l}
u_{1} \\
\ldots \\
u_{n}
\end{array}\right)
$$

From the solution of the linear system for $t \geq t_{0}=0$

$$
z(t)=e^{A(t)}\left(z(0)+\int_{0}^{t} e^{-A \tau} b v(\tau) d \tau\right)
$$

we conclude that a control defined by the pair $(x, u)$ will steer $z_{0}$ to $z=0$ if

$$
\Phi(x) u=-z_{0}
$$




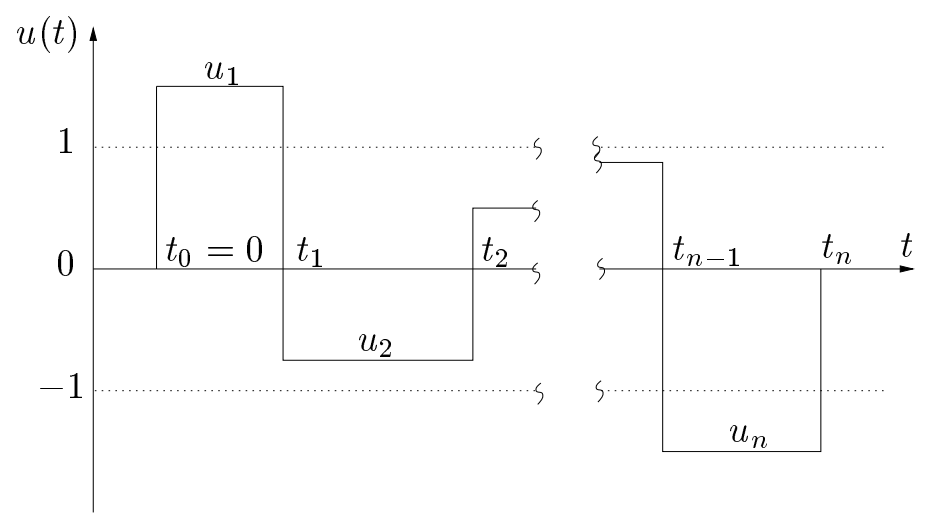

Figure 1: Control sequence.

where the $i$-th column of the matrix $\Phi$ is

$$
\Phi(:, i)(x) \triangleq \int_{t_{i-1}}^{t_{i}} e^{-A \tau} b d \tau=\int_{\sum_{k=0}^{i-1} x_{k}}^{\sum_{k=0}^{i} x_{k}} e^{-A \tau} b d \tau
$$

The optimal vector $\bar{x}$ is the unique solution of (3) when $u=\bar{u}$ is the vector of optimal control values, that is $\left|\bar{u}_{i}\right|=1$ and $u_{i} u_{i+1}=-1$ for $i=1, \ldots, n-$ 1. The equation $\Phi(x) \bar{u}=-z_{0}$ is the nonlinear equation to be solved to determine the optimal control. In contrast, (3) is linear in $u$ and is easily solved for a given $x$. Because the pair $(A, b)$ is controllable, a unique solution $u(x)$ exists for any $x$ in the open positive orthant. A natural class of iterative methods thus consists in updating the time intervals vector $x$ such as to enforce convergence of the corresponding control vector $u(x)$ to a bang-bang sequence of magnitude $\left|u_{i}\right|=1$.

The heuristics considered in (Yastreboff 1969a)-(Yastreboff 1969b) and more recently in (De Doná 2000) is a 'decentralized' adaptation of the vector $x$ : if $\left|u_{i}(x)\right|$ is larger than one, decrease the length of the corresponding time interval $x_{i}$; if $\left|u_{i}(x)\right|$ is smaller than one, increase the length of the corresponding time interval $x_{i}$.

In continuous-time, this heuristics yields the decentralized adaptation

$$
\dot{x}_{i}=f_{i}\left(\left|u_{i}(x)\right|-1\right) x_{i}, i=1, \ldots, n
$$

where $f_{i}$ should be a (smooth) scalar function in the first and third quadrant and only vanish at zero, and where $x_{i}$ is put in factor of $f_{i}$ in order to guarantee the positive invariance of the open positive orthant. Under these conditions the unique equilibrium of $(5)$ in $\mathcal{O}^{+}$is $\bar{x}$, the vector of optimal time intervals.

In discrete-time, the same heuristics yields the decentralized adaptation

$$
x_{i}(k+1)=x_{i}(k)+f_{i}\left(\left|u_{i}(x(k))\right|-1\right) x_{i}(k), i=1, \ldots, n
$$


which is a Euler discretization of (5).

In (Yastreboff 1969b), $f_{i}$ is chosen as

$$
\begin{aligned}
f_{i}\left(\left|u_{i}\right|-1\right)= & q \ln \left|u_{i}\right| & \text { if } & \left|u_{i}(k)\right| \geq 1 \\
& -q \ln \left(2-\left|u_{i}\right|\right) & \text { if } & \left|u_{i}(k)\right|<1
\end{aligned}
$$

where $q>0$ is a constant parameter. In (De Doná 2000), $f_{i}$ is chosen as $f_{i}\left(\left|u_{i}\right|-1\right)=\left|u_{i}\right|^{p}-1$, with $0<p<1$ a constant parameter. Both algorithms were derived independently but it is apparent that they are closely related. Simulation studies in (De Doná 2000) show that both algorithms perform similarly, provided that the respective tuning parameters $p$ and $q$, are properly chosen. Several examples are included in (Yastreboff 1969a)(Yastreboff 1969b), in which the algorithm is compared with other existing methods (Smith 1961, Fadden \& Gilbert 1964, Fadden 1965). Extensive simulations suggest that both the continuous-time algorithm (5) and its discretetime version (6) converge (globally) to the time-optimal solution and that the convergence properties are not very sensitive to the details of the function $f_{i}$, except for its slope at the origin (a higher slope seems to be favorable to the convergence).

\section{An algorithm analysis based on time scale sepa- ration}

The main result of the present paper is to show that the phase portrait of the continuous-time algorithm (5) becomes rather transparent in the simplified situation where $f_{i}$ is linear, yielding the algorithm:

$$
\dot{x}_{i}=p_{i}\left(\left|u_{i}(x)\right|-1\right) x_{i}, \quad i \in\{1, \cdots, n\}, x_{i}(0)>0
$$

With $p_{n}>>p_{n-1}>>\cdots>>p_{1}>0$ positive constants, a time-scale separation can be enforced between the different $x_{i}$ dynamics, as illustrated by

Figure 3. In the fastest time-scale $\tau_{n}=\frac{t}{p_{n}}$, the state $x_{n}$ converges to the manifold $\mathcal{M}_{n}$ defined by $u_{n}=\bar{u}_{n}$. In this manifold, the fastest time-scale is $\tau_{n-1}=\frac{t}{p_{n-1}}$, and the state $x_{n-1}$ converges to the manifold $\mathcal{M}_{n-1}$ defined by $u_{n-1}=\bar{u}_{n-1}$ and $u_{n}=\bar{u}_{n}$. Reducing the dynamics $n$ times eventually leads to the convergence of $x_{1}$ in the slowest time-scale $\tau_{1}=\frac{t}{p_{1}}$ to the manifold $\mathcal{M}_{1}$ defined by $u=\bar{u}$, which reduces to the unique equilibrium point $x=\bar{x}$, as a consequence of the uniqueness of the optimal solution.

Due to space constraints, we will not provide all details of the claim. The crucial result is to establish that the manifolds $\mathcal{M}_{i}(i=n, \cdots, 1)$ define a sequence of nested manifolds of decreasing dimension $i-1$ in a neighborhood of $\bar{x}$. 

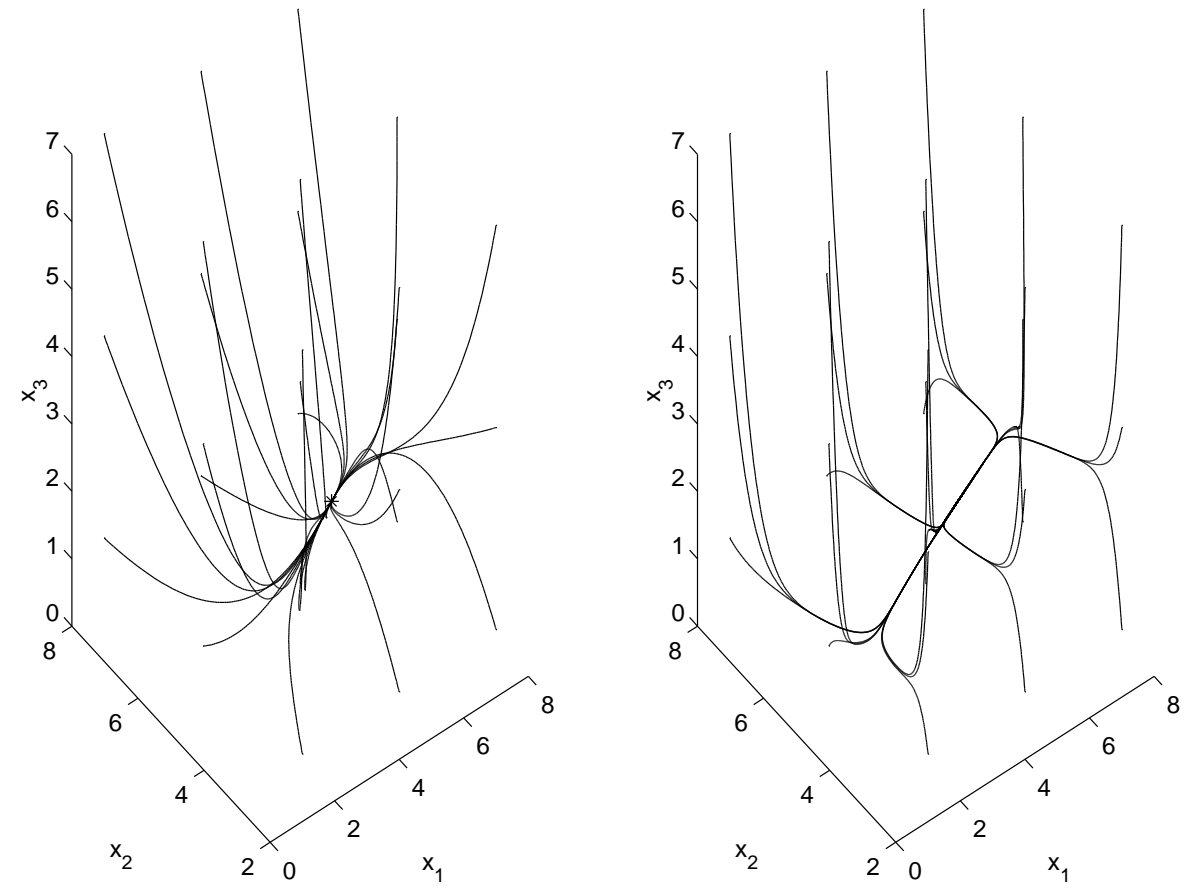

Figure 2: Simulations of (8) for the triple integrator $\dddot{y}=u$. The initial condition in the state-space is $y(0)=\dot{y}(0)=\ddot{y}(0)=2$. The time-scale separation is ensured with, respectively, $p_{3}=2 p_{2}=4 p_{1}=4$ (left) and $p_{3}=10 p_{2}=100 p_{1}=100$ (right) 
Theorem 3.1 Let $u=u(x)$ defined by the steering equation $\Phi(x) u=-z_{0}$ and $x=\bar{x}$ the solution of $\Phi(x) u=-z_{0}$ for $u=\bar{u}$. Then there exists an open neighborhood $\Omega$ of $\bar{x}$ such that the manifold $\mathcal{M}_{l}$ defined by $u_{i}(x)=\bar{u}_{i}$, for $i \in\{l, \cdots, n\}$ satisfy:

i $\operatorname{dim}\left(\mathcal{M}_{l}\right)=l-1$

ii $\{\bar{x}\}=\mathcal{M}_{1} \subset \mathcal{M}_{2} \subset \cdots \subset \mathcal{M}_{n-1} \subset \mathcal{M}_{n} \subset \mathcal{O}^{+}$

iii $\forall x \in \Omega, \mathcal{M}_{l}$ has the explicit representation

$$
\left(\begin{array}{c}
x_{l} \\
\vdots \\
x_{n}
\end{array}\right)=F_{l}\left(x_{1}, \cdots, x_{l-1}\right)
$$

Define the matrix $\mathrm{E}$ of the partial derivatives of $|u|$

$$
e_{i j}=\frac{\partial u_{i}}{\partial x_{j}} \operatorname{sign}\left(u_{i}\right) \quad \text { for } i, j \in\{1, \cdots, n\}
$$

and $E_{l}$, with $1 \leq l \leq n$, as the $(\mathrm{n}-\mathrm{l}+1) \times(\mathrm{n}-\mathrm{l}+1)$ block of $E$ made of the elements $e_{i j}$ for $i, j, \in\{l, \cdots, n\}$. Then the proof of Theorem 3.1 follows from the implicit function theorem provided that the matrix $E_{l}$ has full rank at $x=\bar{x}$ for $l \in\{1, \cdots, n\}$. In fact, we can prove the following stronger property, which will also be used to establish the attractivity of each manifold.

Theorem 3.2 For each $l \in\{1, \cdots, n\}$, and for each $x$ such that $\left(u_{l}, \cdots, u_{n}\right)=$ $\left(\bar{u}_{l}, \cdots, \bar{u}_{n}\right)$, the matrix $E_{l}$ has full rank and

$$
\operatorname{det}\left(E_{l}\right)=(-1)^{n-l+1}\left|\operatorname{det}\left(E_{l}\right)\right|
$$

The proof of Theorem 3.2 relies on linear operations on lines and columns of $E_{l}$ that leave the sign of the determinant unchanged but transforms the matrix into a triangular matrix with negative elements on its diagonal.

Instrumental to these manipulations (see Appendix) are the following two lemmas, the first of which uses the fact that all eigenvalues of $A$ are real, and the second of which rests on optimality principles. 
Lemma 3.1 For each $l \in\{1, \cdots, n\}$, and for each $x$ such that $u_{i} u_{i+1}<0$ for $i \in\{l, \cdots, n-1\}$, each line of $E_{l}$ has its minimum element on the diagonal. Inside a given line, the elements $e_{i k}$ increase with $k$ while staying negative for $k \geq i$, and they decrease with $k$ decreasing $k \leq i$. In other words, the elements of $E_{l}$ satisfy:

- $e_{i k}<0$ for $k \in\{i, \cdots, n\}$;

- $e_{i k}<e_{i k+1}$ for $k \in\{i+1, \cdots, n\}$

- $e_{i k}>e_{i k+1}$ for $k \in\{l, \cdots, i-1\}$

Proof Let $i \geq l$. In order to find $e_{i k}=\frac{\partial u_{i}}{\partial x_{k}} \operatorname{sign}\left(u_{i}\right)$, we differentiate (3) with respect to $x_{k}$, to obtain:

$$
\frac{\partial \Phi}{\partial x_{k}} u+\Phi \frac{\partial u}{\partial x_{k}}=0
$$

Using the expressions:

$$
\begin{aligned}
\frac{\partial \Phi(:, j)}{\partial x_{k}} & =0 & & \text { if } k>j \\
& =e^{-A t_{k}} b & & \text { if } k=j \\
& =-A \Phi(:, j) & & \text { if } k<j
\end{aligned}
$$

(10) becomes:

$$
\Phi \frac{\partial u}{\partial x_{k}}=-e^{-A t_{k}} b u_{k}+\sum_{j=k+1}^{n} A \Phi(:, j) u_{j}
$$

Premultipliying both sides of (11) by $\Phi^{-1}(i,:)$, we obtain:

$$
\frac{\partial u_{i}}{\partial x_{k}}=-\Phi^{-1}(i,:) e^{-A t_{k}} b u_{k}+\sum_{j=k+1}^{n} \Phi^{-1}(i,:) A \Phi(:, j) u_{j}
$$

The scalar function:

$$
f_{i}(t)=\Phi^{-1}(i,:) \int_{0}^{t} e^{-A \tau} b d \tau
$$

satisfies

$$
\begin{array}{lll}
f_{i}(0) & =0 & \\
f_{i}\left(t_{j}\right)=\sum_{m=1}^{j} \Phi^{-1}(i,:) \Phi(:, m)=0 & \text { for } j<i \\
f_{i}\left(t_{j}\right)=\sum_{m=1}^{j} \Phi^{-1}(i,:) \Phi(:, m)=1 & \text { for } j \geq i
\end{array}
$$

It then follows that the derivative $g_{i}(t)=f_{i}^{\prime}(t)=\Phi^{-1}(i,:) e^{-A t} b$ has at least $i-1$ zeros between $t=0$ and $t=t_{i-1}$, and $n-i$ zeros between 
$t=t_{i}$ and $t=t_{n}$. Because the eigenvalues of $A$ are real, the function $g_{i}(t)=\Phi^{-1}(i,:) e^{-A t} b$ has at most $n-1$ zeros (Lee \& Markus 1967), which means that there are no other zeros but the ones exhibited here. A first consequence of this, is that $g_{i}(t)$ has no zero between $t=t_{i-1}$ and $t=t_{i}$. A second one is that $g_{i}\left(t_{j}\right) \neq 0$, and that $g_{i}\left(t_{j}\right)$ alternate sign for $j \leq i-1$ and $j \geq i$, with $g_{i}\left(t_{i-1}\right)$ and $g_{i}\left(t_{i}\right)$ being both positive. These conclusions can be summed up as:

$$
\begin{aligned}
g_{i}\left(t_{j}\right) & =\left|g_{i}\left(t_{j}\right)\right|(-1)^{j-i} & & \text { if } j \geq i \\
& =\left|g_{i}\left(t_{j}\right)\right|(-1)^{i-j-1} & & \text { if } j<i
\end{aligned}
$$

By assumption, the signs of the controls $u_{j}$ alternate for $j \in\{l, \cdots, n\}$, which means that

$$
u_{j}=\left|u_{j}\right| \operatorname{sign}\left(u_{i}\right)(-1)^{j-i} \text { for } j \in\{l, \cdots, n\}
$$

Moreover

$$
\begin{aligned}
\Phi^{-1}(i,:) A \Phi(:, j) u_{j} & =\Phi^{-1}(i,:) A \int_{t_{j-1}}^{t_{j}} e^{-A \tau} b d \tau u_{j} \\
& =-\left(\Phi^{-1}(i,:) e^{-A t_{j}} b-\Phi^{-1}(i,:) e^{-A t_{j-1}} b\right) \\
& =-\left(g_{i}\left(t_{j}\right)-g_{i}\left(t_{j-1}\right)\right) u_{j}
\end{aligned}
$$

which gives, if $n \geq k \geq i \geq l$ :

$$
\begin{aligned}
\frac{\partial u_{i}}{\partial x_{k}=} & -g_{i}\left(t_{k}\right) u_{k}+\sum_{j=k+1}^{n} \Phi^{-1}(i,:) A \Phi(:, j) u_{j} \\
= & -g_{i}\left(t_{k}\right) u_{k}-\sum_{j=k+1}^{n}\left(g_{i}\left(t_{j}\right)-g_{i}\left(t_{j-1}\right)\right) u_{j} \\
= & -\left|g_{i}\left(t_{k}\right)\right|(-1)^{k-i}\left|u_{k}\right| \operatorname{sign}\left(u_{i}\right)(-1)^{k-i} \\
& -\sum_{j=k+1}^{n}\left(\left|g_{i}\left(t_{j}\right)\right|(-1)^{j-i}-\left|g_{i}\left(t_{j-1}\right)\right|(-1)^{j-i-1}\right)\left|u_{j}\right| \operatorname{sign}\left(u_{i}\right)(-1)^{j-i} \\
= & -\left|g_{i}\left(t_{k}\right) u_{k}\right| \operatorname{sign}\left(u_{i}\right)-\sum_{j=k+1}^{n}\left(\left|g_{i}\left(t_{j}\right) u_{j}\right| \operatorname{sign}\left(u_{i}\right)+\left|g_{i}\left(t_{j-1}\right) u_{j}\right| \operatorname{sign}\left(u_{i}\right)\right)
\end{aligned}
$$

It clearly appears that the sign of all the terms of this sum is opposite to the sign of $u_{i}$, which gives:

$$
\frac{\partial u_{i}}{\partial x_{k}} \operatorname{sign}\left(u_{i}\right)=e_{i k}<0 \quad \text { for } n \geq k \geq i \geq l
$$

Moreover, we can see that

$$
\frac{\partial u_{i}}{\partial x_{k}}=\frac{\partial u_{i}}{\partial x_{k+1}}-\left|g_{i}\left(t_{k}\right) u_{k}\right| \operatorname{sign}\left(u_{i}\right)-\left|g_{i}\left(t_{k}\right) u_{k+1}\right| \operatorname{sign}\left(u_{i}\right)
$$

and, multiplying both sides by $\operatorname{sign}\left(u_{i}\right)$ :

$$
e_{i k}<e_{i k+1} \quad \text { for } n \geq k \geq i \geq l
$$

When $l \leq k \leq i-1$,

$$
\begin{aligned}
\frac{\partial u_{i}}{\partial x_{k}}= & \frac{\partial u_{i}}{\partial x_{k+1}}-g_{i}\left(t_{k}\right) u_{k}+g_{i}\left(t_{k}\right) u_{k+1} \\
= & \frac{\partial u_{i}}{\partial x_{k+1}}-\left|g_{i}\left(t_{k}\right)\right|(-1)^{i-k-1}\left|u_{k}\right| \operatorname{sign}\left(u_{i}\right)(-1)^{i-k} \\
& +\left|g_{i}\left(t_{k}\right)\right|(-1)^{i-k-1}\left|u_{k+1}\right| \operatorname{sign}\left(u_{i}\right)(-1)^{i-k-1} \\
= & \frac{\partial u_{i}}{\partial x_{k+1}}+\left|g_{i}\left(t_{k}\right)\right|\left(\left|u_{k}\right|+\left|u_{k+1}\right|\right) \operatorname{sign}\left(u_{i}\right)
\end{aligned}
$$


and, multiplying both sides by $\operatorname{sign}\left(u_{i}\right)$ :

$$
e_{i k}>e_{i k+1}
$$

Lemma 3.2 For each $l \in\{1, \cdots, n\}$, and for each $x^{0}$ such that $\left(u_{l}, \cdots, u_{n}\right)=$ $\left(\bar{u}_{l}, \cdots, \bar{u}_{n}\right)$, there exists a positive vector $\alpha^{l} \geq 0$ such that

$$
E_{l}^{T} \alpha^{l}=\left(\begin{array}{c}
-1 \\
-1 \\
\vdots \\
-1
\end{array}\right)
$$

Proof: The control law defined by the sequence of time intervals $\left(x_{n}^{0}, \cdots, x_{l}^{0}\right)$ and control values $\left(\bar{u}_{n}, \cdots, \bar{u}_{l}\right)$ is the time-optimal solution of the reversed time system

$$
\dot{z}=-A z-b v \quad|v| \leq 1
$$

with initial condition $z(0)=0$ and as target set the affine subspace

$$
\Pi=e^{A t_{l-1}} z_{0}+\operatorname{span}\left\{e^{A t_{l-1}} \Phi(:, 1), \cdots, e^{A t_{l-1}} \Phi(:, l-1)\right\}
$$

This follows from the application of the maximum principle (Pontryagin et al. 1962).

Variations of $\left(x_{l}^{0}, \cdots, x_{n}^{0}\right)$, with fixed $\left(x_{1}, \cdots, x_{l-1}\right)$ give other solution to the control of the unconstrained reversed time system from $z=0$ to $\Pi$. By optimality of $x^{0}$, a decrease of the total time $\sum_{j=l}^{n} x_{j}$ implies a constraint violation for at least one of the $u_{k}(k \geq l)$.

As a consequence, any control variation $\partial u_{k}$ that satisfies $\operatorname{sign}\left(u_{k}\right) \partial u_{k} \leq$ 0 for $k \in\{l, \cdots, n\}$ will impose a time variation $\sum_{j=l}^{n} x_{j} \geq 0$. This means that $M=0$ is the optimal solution of

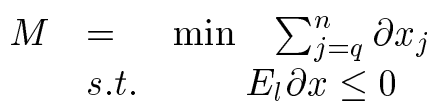

where we have used the relation $\partial u=E_{l} \partial x$ (with $\partial u=\left(\partial u_{l}, \cdots, \partial u_{n}\right)^{T}$, and $\left.\partial x=\left(\partial x_{l}, \cdots, \partial x_{n}\right)^{T}\right)$. The dual formulation of (13) is

$$
\begin{array}{cc}
M= & \max \quad 0 \\
\text { s.t. } & E_{l}^{T} \alpha^{l}=\left(\begin{array}{c}
-1 \\
-1 \\
\vdots \\
-1
\end{array}\right) \\
& \alpha^{l} \geq 0
\end{array}
$$


which proves the existence of $\alpha^{l}$ such that (12) holds.

We now return to the behavior of solutions of (8), assuming $n$ different time scales $\tau_{i}=\epsilon_{i} t=\frac{t}{p_{i}}$. A singular perturbation argument ensures the existence of $n$ nested invariant manifold $\mathcal{M}_{l}^{\epsilon_{l}}(l \in\{1, \cdots, n\})$, each $\mathcal{M}_{l}^{\epsilon_{l}}$ being in a $\mathcal{O} \epsilon_{l}$ neighborhood of the manifold $\mathcal{M}_{l}$.

Solutions in $\mathcal{O}^{+}$sequentially converge to $\mathcal{M}_{n}^{\epsilon_{n}}$, then to $\mathcal{M}_{n-1}^{\epsilon_{n-1}}$, up to the convergence to the equilibrium $\bar{x}=\mathcal{M}_{1}$. For an initial condition $x \in \mathcal{M}_{l+1}^{\epsilon_{l+1}}$, the convergence to $\mathcal{M}_{l}^{\epsilon_{l}}$ is dictated by the scalar equation

$$
\dot{x}_{l}=p_{l}\left(\left|u_{l}\right|-1\right) x_{l}
$$

which has an isolated equilibrium $\tilde{x}$ determined by $\left|u_{l}\right|=1$. Attractivity of this equilibrium is ensured if

$$
\frac{d \dot{x}_{l}}{d x_{l}}=p_{l} x_{l} \frac{d\left|u_{l}\right|}{d x_{l}}<0
$$

An explicit calculation shows that

$$
\frac{d\left|u_{l}\right|}{d x_{l}}=\frac{\operatorname{det}\left(E_{l}\right)}{\operatorname{det}\left(E_{l+1}\right)}<0
$$

which is negative by Theorem 3.2 .

\section{Conclusions}

In this paper we have presented a numerical algorithm for the computation of the time-optimal control solution for linear systems with input constraints. The algorithms considered in this paper include a continuous-time version that is amenable to convergence analysis and two discrete-time analogs that have been previously proposed in the literature without a proof of convergence. The reported time-scale analysis of the continuous-time algorithm constitutes the main contribution of this paper.

\section{Appendix}

Proof of Theorem 3.2 Defining the vector $\alpha^{l}$ given in Lemma 3.2 as $\alpha^{l}=\left(\alpha_{l}^{l}, \cdots, \alpha_{n}^{l}\right)^{T}$, we first show that $\alpha_{l}^{l}>0$. By contradiction, if $\alpha_{l}^{l}=0$, we have:

$$
\sum_{j=l+1}^{n} \alpha_{j}^{l} e_{j l}=\sum_{j=l+1}^{n} \alpha_{j}^{l} e_{j l+1}=-1
$$

which is not possible because $\alpha_{j}^{l} \geq 0$ and $e_{j l}>e_{j l+1}$ for $j \in\{l+1, \cdots, n\}$ (see Lemma 3.1). 
Denote the lines of $E_{l}$ by $E_{l}(l,:), \cdots, E_{l}(n,:)$. The sign of the determinant of $E_{l}$ is unchanged when $E_{l}(l,:)$ is replaced by $\sum_{j=l}^{n} \alpha_{j}^{l} E_{l}(j,:)$ because the determinant is multiplied by $\alpha_{l}^{l}$. The first line of $E_{l}$ is then replaced by $(-1, \cdots,-1)$.

We iterate this operation on the submatrices $E_{i}$ of $E_{l}(\mathrm{i}>\mathrm{l})$ : for instance, we replace $E_{i}(i,:)$ by $\sum_{j=i}^{n} \alpha_{j}^{i} E_{i}(j,:)$ in order to have $(-1, \cdots,-1)$ on the first line. Applying the same transformation to $E_{l}(i,:)$ yields

$$
\sum_{j=i}^{n} \alpha_{j}^{i} E_{l}(j,:)=\left(\times, \times, \cdots, \times, f_{i, i-1},-1,-1, \cdots,-1\right)
$$

We note that $f_{i, i-1}=\sum_{j=i}^{n} \alpha_{j}^{i} e_{j i-1}>\sum_{j=i}^{n} \alpha_{j}^{i} e_{j i}=-1$, where the inequality comes from Lemma 3.1. Ultimately $E_{l}$ is transformed into:

$$
F_{l}=\left(\begin{array}{cccccc}
-1 & -1 & \cdots & -1 & -1 & -1 \\
f_{l+1, l} & -1 & \cdots & -1 & -1 & -1 \\
\times & f_{l+2, l+1} & \cdots & -1 & -1 & -1 \\
& \vdots & \vdots & & & \vdots \\
\times & \times & \cdots & f_{n-1, n-2} & -1 & -1 \\
e_{n, l} & e_{n, l+1} & \cdots & e_{n, n-2} & e_{n, n-1} & e_{n, n}
\end{array}\right)
$$

where

$$
\operatorname{det}\left(F_{l}\right)=\operatorname{det}\left(E_{l}\right) \prod_{j=l}^{n} \alpha_{j}^{j}
$$

Finally, replacing $F_{:, i}^{l}$ by $F_{:, i}^{l}-F_{:, i-1}^{l}$ for $i=n, \cdots, l+1$, we do not change the determinant and the matrix becomes:

$$
G_{l}=\left(\begin{array}{cccccc}
-1 & 0 & \cdots & 0 & 0 & 0 \\
\times & -1-f_{l+1, l} & \cdots & 0 & 0 & 0 \\
\times & \times & \cdots & 0 & 0 & 0 \\
\vdots & & \vdots & & & \vdots \\
\times & \times & \cdots & \times & -1-f_{n-1, n-2} & 0 \\
\times & \times & \cdots & \times & \times & e_{n, n}-e_{n, n-1}
\end{array}\right)
$$

whose diagonal elements are all negative. Hence the result is proven.

\section{References}

Athans, M., Falb, P.L. (1966), Optimal Control. An introduction to the theory and its applications, Mc Graw-Hill, New York.

Bellman, R. (1957), Dynamic Programming, Princeton University Press. 
Bellman, R., Glicksberg, I., Gross, O. (1956), "On the bang-bang control problem," Quarterly of Applied Mathematics, 14, 11-18.

De Doná, J.A. (2000), "Input Constrained Linear Control," PhD. thesis, The University of Newcastle, Australia.

Desoer, C.A. (1959), "The bang-bang servo problem treated by variational techniques," Information and Control, 2, pp. 333-348.

Fadden, E.J. (1965), Computational aspects of a class of optimal control problems, Technical Report ORA Project 06181, University of Michigan, Ann Arbor.

Fadden, E.J., Gilbert E.G. (1964), Computational aspects of the time optimal control problem, in A.V. Balakrishnan \& L.W. Neustadt, eds, "Computing Methods in Optimization Problems," Academic Press, New York.

Gilbert E.G. (1964), "The application of hybrid computers to the iterative solution of optimal control problems, in A.V. Balakrishnan \& L.W. Neustadt, eds, "Computing Methods in Optimization Problems," Academic Press, New York, pp. 261-284.

Ho, Y.C. (1962), "A successive approximation technique for optimal control systems subject to input saturation," Journal of Basic Engineering, Transactions of ASME, 84D, 33-40.

Lee, E.B., Markus, L. (1967), "Foundations of Optimal Control Theory," John Wiley \& Sons, Inc., New York.

Mayne, D.Q., Rawlings, J.B., Rao, C.V., Schockaert, P.O.M. (2000), "Constrained model predictive control: stability and optimality," Automatica, 36, pp. 789-814.

Neustadt, L.W. (1960), "Synthesizing time-optimal control systems," Journal of Mathematical Analysis and Applications, 1, pp. 464-493.

Pontryagin, L.S., Boltyanskii, V., Gamkrelidze, R., Mischenko, E. (1962), "The Mathematical Theory of Optimal Processes", Interscience Publishers, Inc., New York.

Smith, F.B. (1961), "Time-optimal control of higher-order systems," IRE Trans. on Automatic Control, 6, pp. 16-21.

Sontag, E.D. (1998), "Mathematical Control Theory: Deterministic Finite Dimensional Systems", second edition, Springer, New York. 
Yastreboff, M.M. (1969), "Investigation of Several Synthesis Procedures for On-Line Time Optimal Control," PhD. thesis, The University of New South Wales, Australia.

Yastreboff, M.M. (1969), "Synthesis of time-optimal control by time interval adjustment," IEEE Trans. on Automatic Control, 14, pp. 707-710 . 